\title{
RELAÇÃO ENTRE BRUXISMO E O GRAU DE SINTOMATOLOGIA DE DISFUNÇÃO TEMPOROMANDIBULAR
}

\section{The relationship between the bruxism and the severity of symptoms in the temporomandibular disorder}

\author{
Cíntia Corrêa Blini ${ }^{(1)}$, Marcela Forgiarini Morisso (2), \\ Geovana de Paula Bolzan ${ }^{(3)}$, Ana Maria Toniolo da Silva ${ }^{(4)}$
}

\begin{abstract}
RESUMO
Objetivo: verificar a ocorrência de bruxismo em sujeitos adultos do sexo feminino com queixa de disfunção temporomandibular e sua relação com o grau de sintomatologia da disfunção. Métodos: participaram deste estudo 28 mulheres na faixa etária de 19 a 56 anos, que apresentavam sintomas de disfunção temporomandibular e que não haviam realizado tratamento anterior. Todas responderam o questionário de Índice Anamnésico proposto por Fonseca et al (1994), o qual possibilita a classificação do grau de sintomatologia de disfunção temporomandibular e verificação da queixa do hábito parafuncional bruxismo; e realizaram uma avaliação odontológica, constituída de exame da musculatura mastigatória, por meio de palpação digital intra e extra-oral, inspeção das articulações temporomandibulares e exame dental. Os resultados foram analisados descritivamente e, para verificar a relação entre o grau de severidade da disfunção temporomandibular com a ocorrência de bruxismo, foram realizados o Teste de Independência do Qui-quadrado e o Teste Exato de Fisher, ambos ao nível de significância de 5\%. Resultados: verificou-se que o bruxismo esteve presente em $50 \%$ dos casos de disfunção temporomandibular. Não houve relação entre o bruxismo e o grau de sintomatologia de disfunção temporomandibular, estabelecido pelo Índice Anamnésico. Conclusão: os resultados deste estudo sugerem que sujeitos com sintomas de disfunção temporomandibular devem ser questionados e avaliados quanto à presença de bruxismo, independentemente do grau de sintomatologia da disfunção. Assim como deve ser realizado diagnóstico e tratamento do bruxismo em sujeitos assintomáticos de disfunção temporomandibular como forma de prevenir o desenvolvimento de lesões nas articulações temporomandibulares e demais estruturas do sistema estomatognático.
\end{abstract}

DESCRITORES: Bruxismo; Dor Facial; Síndrome da Disfunção da Articulação Temporomandibular

(1) Fonoaudióloga da Secretaria Municipal de Saúde e Centro de Referência em Saúde do Trabalhador, CEREST, Palmeira das Missões, RS, Brasil.

(2) Fonoaudióloga da Associação dos Pais e Amigos do Excepcional, APAE, Candelária, RS, Brasil; Mestre em Distúrbios da Comunicação Humana pela Universidade Federal de Santa Maria.

(3) Fonoaudióloga; Mestranda em Distúrbios da Comunicação Humana na Universidade Federal de Santa Maria, UFSM, Santa Maria, RS, Brasil.

(4) Fonoaudióloga; Professora Associada do Departamento de Fonoaudiologia da Universidade Federal de Santa Maria, UFSM, Santa Maria, RS, Brasil; Doutora em Ciências dos Distúrbios da Comunicação Humana pela Universidade Federal de São Paulo.

Conflito de interesses: inexistente

\section{INTRODUÇÃO}

O bruxismo é definido como o ato de ranger ou apertar os dentes de forma subconsciente ou parafuncional. Este hábito costuma ocorrer durante o sono, porém pode ocorrer também durante vigília. $\mathrm{O}$ aumento de atividade da musculatura mastigatória que ocorre nos momentos de bruxismo tende a afetar os dentes e suas estruturas de suporte, o que é evidenciado por desgaste e mobilidade dentária ${ }^{1}$. A severidade das alterações causadas pelo bruxismo varia conforme a resistência das estruturas atingidas, o tempo de existência, sua regularidade e o estado geral do sujeito afetado ${ }^{2}$. 
No ranger de dentes, mais comum durante 0 sono, predominam os movimentos de deslizamento das superfícies oclusais em contato excêntrico, o que favorece o desgaste dentário ${ }^{2}$. No apertamento dentário, no entanto, ocorre aumento da tensão em oclusão cêntrica por contração isométrica dos músculos elevadores da mandíbula, o que pode levar ao surgimento de dor, fadiga e hipertrofia da musculatura mastigatória, além de prejudicar as estruturas de suporte dos dentes. O apertamento dentário ocorre geralmente quando o sujeito está concentrado em alguma tarefa ou desempenhando algum trabalho que exija muito esforço físico, embora também possa ocorrer durante o sono ${ }^{2,3}$.

A relação entre bruxismo e disfunção temporomandibular (DTM) vem sendo estudada por diversos autores devido aos prejuízos que este hábito parafuncional pode acarretar ao sistema estomatognático como um todo ${ }^{4}$. Os movimentos mandibulares não funcionais que ocorrem no bruxismo resultam em uma solicitação anormal dos músculos da mastigação, que em estado de hiperfunção, podem apresentar sintomatologia dolorosa e diminuição de sua coordenação ${ }^{5}$, o que faz com que este hábito seja considerado importante fator contribuinte de alterações na articulação temporomandibular (ATM) 3,4,6-9. $^{3}$.

As alterações que acometem a ATM têm etiologia multifatorial e manifestam-se por sintomatologia dolorosa na região orofacial e cervical, limitações dos movimentos mandibulares e das funções estomatognáticas a eles associadas ${ }^{1,10}$. Estudos recentes evidenciaram a prevalência de DTM no sexo feminino sobre o masculino ${ }^{11-14} \mathrm{e}$ destacaram que os aspectos emocionais podem influenciar tanto a ocorrência de DTMs ${ }^{12,14-16}$ quanto de bruxismo 4,17,18.

A avaliação das DTMs é, em geral, realizada por equipe multidisciplinar, por meio de entrevista criteriosa para levantamento dos sintomas, circunstâncias e impressões do sujeito em relação à queixa, seguida de exame clínico específico, que possibilitará o diagnóstico e identificação de interferências determinantes ${ }^{10}$.

Visando identificar os sintomas de DTM de forma breve, Fonseca et al (1994) ${ }^{19}$ propuseram o Índice Anamnésico, um questionário em língua portuguesa que investiga sintomas de DTM e, quando presentes, classifica-os em graus de severidade. Este instrumento tem sua utilização limitada por não ser uma ferramenta para diagnóstico de DTM, como é o caso do RDC/TMD, Critérios de Diagnóstico para Pesquisa das Disfunções Temporomandibulares ${ }^{20}$, largamente utilizado. Porém, o Índice Anamnésico pode auxiliar na verificação inicial de sintomas de DTM, indicando a necessidade de um exame clínico criterioso, que avalie os sinais clínicos da disfunção, além de auxiliar o clínico a determinar a severidade dos sintomas de DTM por meio de perguntas simples.

Dessa forma, o objetivo deste estudo foi verificar a ocorrência de bruxismo em sujeitos adultos do sexo feminino com queixa DTM e sua relação com o grau de sintomatologia da disfunção, conforme o Índice Anamnésico.

\section{MÉTODOS}

Este estudo é de caráter exploratório, quantitativo, contemporâneo e transversal. Participaram do mesmo, 28 mulheres com idades entre 19 e 56 anos, que apresentavam sintomatologia de disfunção temporomandibular. A amostra foi selecionada a partir da procura das participantes por tratamento em clínicas-escolas de cursos de Odontologia de duas universidades. Foram incluídas na pesquisa, apenas as voluntárias que tiveram diagnóstico clínico de sintomatologia de disfunção temporomandibular (DTM) e que não haviam realizado tratamento odontológico, fisioterápico e/ou fonoaudiológico para esta disfunção. Foram excluídas do estudo as que apresentaram histórico de cirurgia bucomaxilofacial e/ou que utilizavam prótese dentária, a fim de evitar possíveis interferências decorrentes de tais condições.

Inicialmente as participantes responderam o questionário de Índice Anamnésico, que consiste de 10 perguntas referentes aos principais sintomas de disfunção temporomandibular. As perguntas investigam presença de dor; hábitos parafuncionais, tais como o bruxismo; limitação de movimentos mandibulares; presença de ruídos articulares; percepção subjetiva de má-oclusão e tensão emocional. Cada questão do questionário foi lida em voz alta para a participante, individualmente, e esta deveria marcar no formulário apenas uma das três repostas possíveis: "sim", "não" e "às vezes". Para cada resposta foram atribuídas pontuações ${ }^{19}$, que variaram de 0 a 10 pontos, sendo que 0 correspondeu à marcação "não"; 5 correspondeu à marcação "às vezes" e 10 à marcação "sim". A partir do índice anamnésico obtido com a análise das respostas, definiu-se o grau de severidade da sintomatologia da disfunção temporomandibular de cada voluntária: de 20 a 40 pontos disfunção de grau leve; de 45 a 65 pontos disfunção de grau moderado; de 70 a 100 pontos disfunção de grau severo. As participantes que obtiveram índice abaixo de 15 pontos foram excluídas da amostra, uma vez que este resultado sugere ausência de alteração na articulação temporomandibular ${ }^{19}$.

Após responderem o questionário, as participantes que se enquadram nos critérios do estudo

Rev. CEFAC, São Paulo 
foram submetidas a exame clínico da musculatura mastigatória, das ATMs e dos dentes, conforme Okeson (2000) ${ }^{1}$, realizado por cirurgião dentista. A avaliação muscular consistiu na palpação digital com uma única pressão firme, com duração de um a dois segundos, sobre os principais músculos da mastigação (temporal anterior, médio e posterior, masseter superficial e profundo, pterigóideo medial, tendão do temporal) e sobre os músculos que fornecem apoio secundário, como os do pescoço (esternocleidomastóideo, trapézio, músculos cervicais posteriores). Durante a palpação as voluntárias foram questionadas quanto à sensação de dor ou desconforto, pois a sensação dolorosa à palpação destes músculos caracteriza sinal e/ou sintoma de disfunção temporomandibular ${ }^{1}$.

A inspeção das ATMs também foi realizada por meio de palpação digital tanto no repouso quanto em movimento de abertura, fechamento e lateralização mandibular, sendo verificada a ocorrência de sons articulares (estalido ou creptação), sem uso de estetoscópio, desvios e/ ou sensação de dor ${ }^{1}$.

No exame dental foi verificada a condição oclusal das voluntárias em todas as posições e movimentos mandibulares possíveis: relação cêntrica, posição de intercuspidação, movimento protrusivo e movimentos laterotrusivos direito e esquerdo. As estruturas dentárias foram avaliadas com relação a qualquer colapso que pudesse sugerir distúrbio funcional, do tipo bruxismo. Neste sentido, foram investigadas criteriosamente a presença de mobilidade dental maior que $0,5 \mathrm{~mm}$ e facetas de desgaste na superifície incisal dos dentes anteriores e na região oclusal dos dentes posteriores, não causadas pela atrição fisiológica da mastigação ${ }^{1}$. Além disso, durante a avaliação odontológica as participantes foram questionadas quanto a hábitos parafuncionais, para confirmação dessa questão do questionário de Índice Anamnésico.

Com a análise do exame clínico odontológico confirmou-se a presença de sinais clínicos de DTM em todas as participantes, cujo grau de sintomatologia foi estabelecido pelo Índice Anamnésico, e foram diagnosticadas as que apresentavam bruxismo. Considerou-se bruxistas aquelas que apresentaram presença generalizada e/ou localizada de facetas de desgaste dental alinhadas e/ ou mobilidade dentária maior que $0,5 \mathrm{~mm}{ }^{1}$ e presença de pelo menos um dos sinais ou sintomas de bruxismo: auto-relato de ranger ou apertar os dentes durante o sono e/ou vigília, sensibilidade dolorosa à palpação dos músculos mastigatórios, desconforto mandibular ao despertar ${ }^{4}$.

Todas as voluntárias foram submetidas a avaliações somente após a assinatura do termo de consentimento livre e esclarecido, aprovado juntamente com o projeto de pesquisa pelo Comitê de Ética em Pesquisa da instituição de origem sob o número 116/2004.

Os resultados foram analisados descritivamente e para verificar a relação entre o grau de sintomatologia da disfunção temporomandibular com a ocorrência de bruxismo foi realizado o Teste de Independência do Qui-quadrado com base na Tabela 1. Porém, o resultado deste teste ficou comprometido, pois ocorreram mais de $20 \%$ das frequências esperadas inferiores a 5\%. Em vista de tal comprometimento, optou-se por utilizar uma tabela $2 \times 2$, em que os graus leve e moderado de DTM foram agrupados em uma única categoria. A escolha pela união destas duas variáveis ocorreu por acreditarse haver maior semelhança entre os sintomas presentes nestes graus do que entre as outras combinações possíveis. Dessa forma, realizou-se o Teste Exato de Fisher com base na Tabela 2. Para ambos os testes adotou-se o nível de significância de 5\% $(p<0,05)$.

\section{RESULTADOS}

A Tabela 1 mostra as frequências observadas para grau de sintomatologia de DTM e bruxismo. Verificou-se que o bruxismo ocorreu em $50 \%$ dos sujeitos sintomáticos de DTM, sendo mais fre-

Tabela 1 - Relação entre os graus leve, moderado e severo de DTM e bruxismo

\begin{tabular}{cccccc}
\hline \multirow{2}{*}{ DTM - grau de sintomatologia } & \multicolumn{5}{c}{ Bruxismo } \\
\cline { 2 - 5 } & \multicolumn{3}{c}{ Presente } & \multicolumn{3}{c}{ Ausente } & \\
\cline { 2 - 5 } & $\mathbf{N}$ & $\%$ & $\mathbf{n}$ & $\%$ \\
\hline Leve & 3 & 10,7 & 7 & 25,0 & \\
Moderado & 4 & 14,3 & 5 & 17,9 & $\mathrm{p}=0,106^{\star}$ \\
Severo & 7 & 25,0 & 2 & 7,1 & \\
\hline Total & 14 & 50 & 14 & 50 \\
\hline
\end{tabular}

Legenda: *Teste Qui-Quadrado: $p<0,05$; DTM - Disfunção temporomandibular 
quente nos sujeitos com grau severo de sintomatologia (25\%), seguido pelo grau moderado (14,3\%) e pelo grau leve (10,7\%). A distribuição da frequência do bruxismo em cada grau de sintomatologia de DTM pode ser verificado nas Figuras 1, 2 e 3.

Por meio do teste de Independência do Qui-quadrado (Tabela 1) verificou-se que não houve rela- ção entre o grau de sintomatologia de DTM, leve, moderado e severo, e a ocorrência de bruxismo $(p=0,106)$.

Com a realização do Teste Exato de Fisher, com base na Tabela 2, verificou-se que não houve relação entre o grau de sintomatologia de DTM, levemoderado e severo, e bruxismo $(p=0,103)$.

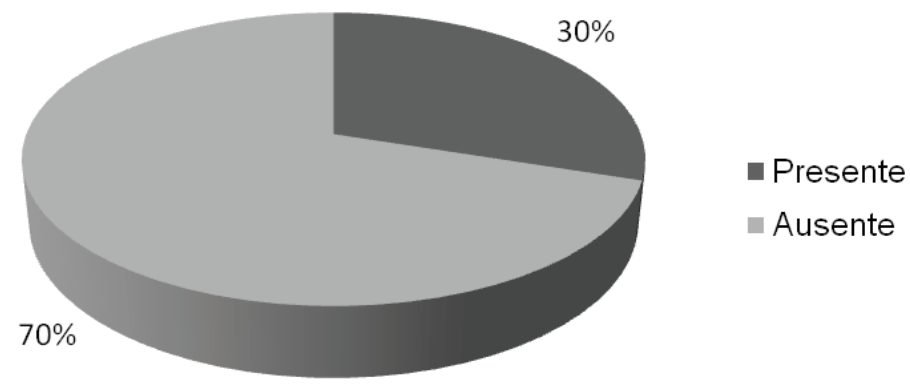

Figura 1 - Presença de bruxismo no grau leve de sintomatologia de DTM

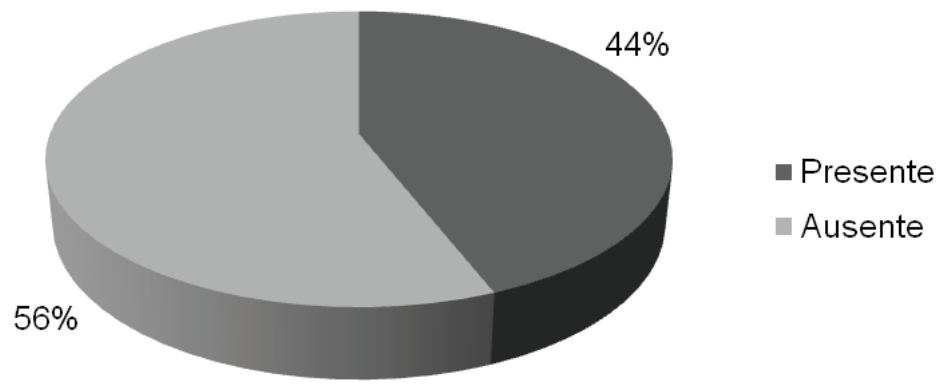

Figura 2 - Presença de bruxismo no grau moderado de sintomatologia da DTM

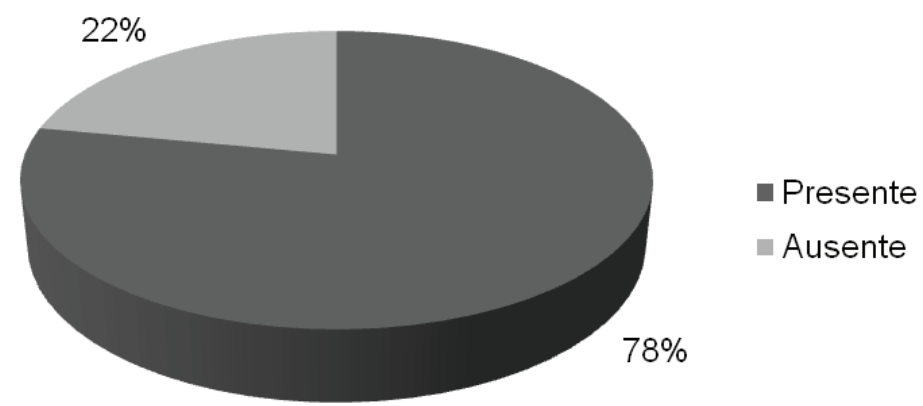

Figura 3 - Presença de bruxismo no grau severo de sintomatologia da DTM

Tabela 2 - Relação entre os graus leve-moderado e severo de DTM e bruxismo

\begin{tabular}{|c|c|c|c|c|c|}
\hline \multirow{3}{*}{ DTM - grau de sintomatologia } & \multicolumn{4}{|c|}{ Bruxismo } & \\
\hline & \multicolumn{2}{|c|}{ Presente } & \multicolumn{2}{|c|}{ Ausente } & \\
\hline & $\mathbf{N}$ & $\%$ & $\mathbf{n}$ & $\%$ & \\
\hline Leve - Moderado & 7 & 25,0 & 12 & 42,9 & \multirow{2}{*}{$p=0,103$} \\
\hline Severo & 7 & 25,0 & 2 & 7,1 & \\
\hline Total & 14 & 50 & 14 & 50 & \\
\hline
\end{tabular}

Legenda: *Teste Exato de Fisher. $\mathrm{p}<0,05$; DTM - Disfunção temporomandibular 


\section{DISCUSSÃO}

Os resultados deste estudo evidenciaram presença de bruxismo em $50 \%$ das participantes sintomáticas de DTM, concordando com a literatura que verificou frequente ocorrência de bruxismo em sujeitos de ambos os sexos com disfunção temporomandibular ${ }^{4,8,21}$. Uma pesquisa realizada com 177 adultos jovens verificou prevalência de bruxismo em $53,9 \%$ dos sujeitos com DTM e em $37,6 \%$ dos sujeitos sem DTM, com presença de associação significante entre DTM e bruxismo ${ }^{4}$. Outra pesquisa recentemente desenvolvida com 182 sujeitos com diagnóstico de DTM verificou presença de bruxismo em $76,9 \%$ da amostra, sendo mais frequente o bruxismo durante vigília, embora a associação entre bruxismo diurno e noturno tenha ocorrido em $43,4 \%$ dos casos ${ }^{21}$. No presente estudo não houve diferenciação quanto ao tipo de bruxismo, nem quanto ao momento em que ocorre.

Os danos que o bruxismo pode acarretar às articulações temporomandibulares e ao sistema estomatognático como um todo decorrem da contração muscular por tempo prolongado, que ocorre durante os momentos de atividade parafuncional, e da força aplicada sobre a superfície oclusal, em torno de seis vezes maior do que a que ocorre nos movimentos fisiológicos ${ }^{2}$.

Esta associação entre bruxismo e DTM talvez possa ser explicada pela presença de aspectos emocionais como fator desencadeante e mantenedor do hábito parafuncional e conseqüentemente da sintomatologia de DTM 4,17,18. A relação entre estresse emocional e DTM foi evidenciada em estudo com sujeitos adultos de ambos os sexos, e que utilizou também o Índice Anamnésico para identificar os sujeitos sintomáticos desta disfunção ${ }^{16}$. A literatura salienta que a dor na musculatura mastigatória de sujeitos com DTM parece estar mais relacionada a estresse emocional do que com a ocorrência de hábitos parafuncionais ${ }^{22}$, porém acredita-se que o estresse determine a ocorrência do hábito parafuncional, estando ambos, portanto, de forma associada, contribuindo para o surgimento e/ou manutenção da dor.

A prevalência da DTM no sexo feminino referenciada na literatura ${ }^{11-14}$ é que motivou a escolha deste sexo para a realização do presente estudo. Considerando a relevância dos aspectos emocionais na ocorrência das disfunções estudadas, acredita-se que a maior ocorrência de DTM no sexo feminino possa ter relação com as condições emocionais desfavoráveis, que tendem a ocorrer com maior frequência entre as mulheres ${ }^{23}$. A relação positiva entre DTM, sexo feminino e estresse emocional foi evidenciada nos estudos de Manfred et al (2006) ${ }^{12}$. Porém, a associação entre DTM e depressão foi verificada atualmente em ambos os sexos ${ }^{14}$. Estes achados reforçam a importância da equipe multidisciplinar na avaliação e tratamento de DTM, dor orofacial e bruxismo, sendo essencial a investigação de aspectos emocionais, que possam contribuir para o agravamento do quadro e para o insucesso de intervenções terapêu-ticas ${ }^{15,18}$.

A maior parte das participantes deste estudo que tiveram diagnóstico de bruxismo apresentou grau severo de sintomatologia de DTM, seguido pelos graus moderado e leve. No entanto não foi verificada relação entre bruxismo e grau de sintomatologia de DTM. Tal achado vai de encontro com a literatura ${ }^{24}$ que estudou por meio de questionário a presença de bruxismo em 276 sujeitos com DTM e constatou que a presença desta parafunção correlaciona-se de maneira linear com o grau de severidade da disfunção. Acredita-se que a discordância entre tais achados possa ser explicada pelo tamanho da amostra do presente estudo. Novas investigações no sentido de verificar uma possível associação entre grau de sintomatologia de DTM e bruxismo devem ser realizadas com amostra mais expressiva.

Comumente sujeitos que apresentam bruxismo, sobretudo durante o sono, desconhecem a ocorrência deste hábito, que é relatado por familiares devido aos sons realizados 9 . Devido ao desconhecimento da parafunção, o diagnóstico clínico de bruxismo muitas vezes ocorre quando o quadro clínico já tomou proporções avança-das ${ }^{2}$. É imprescindível, portanto, que os profissionais da área da saúde procurem identificar esse hábito parafuncional por meio de questionamentos e exame clínico, para que seja tratado como fator de risco para o desenvolvimento das diferentes formas de DTM ${ }^{3,21}$. Assim como também deve ser investigada a ocorrência de bruxismo em sujeitos que apresentam sintomatologia de DTM, independente do grau, a fim de prevenir o agravamento do quadro.

\section{CONCLUSÃO}

A ocorrência de bruxismo foi verificada em $50 \%$ das participantes com queixa de DTM. Não houve relação entre este hábito parafuncional e o grau de sintomatologia de DTM, estabelecido pelo Índice Anamnésico. Tais achados sugerem que sujeitos com sintomas de disfunção temporomandibular devem ser questionados e avaliados quanto à presença de bruxismo, independentemente do grau de sintomatologia da disfunção. Assim como devem ser realizados o diagnóstico e tratamento do bruxismo em sujeitos assintomáticos de DTM como forma de prevenir o desenvolvimento de lesões nas ATMs e demais estruturas do sistema estomatognático. 


\section{ABSTRACT}

Purpose: to check the occurrence of bruxism in female gender adult subject, with temporomandibular jaw dysfunction and its association with the level of temporomandibular symptoms. Methods: 28 women, with ages between 19 and 56 years, who had temporomandibular jaw dysfunction and had not been submitted to a preview treatment, made part of this study. All subjects answered a questionnaire written by Fonseca et al (1994) entitled Anamnesic Index, that enabled us to classify temporomandibular jaw symptoms level and also to check the symptoms of this oral parafunction behavioral bruxism; the subjects were submitted an odontological evaluation, that was composed by an exam of masticatory muscles, though the digital touch in the oral and extra-oral muscles, examination in the temporomandibular joint and a teeth examination. The results were analyzed in a descriptive form, and in order to check the relationship between the severity level of temporomandibular dysfunction with the bruxism occurrence Independent Square-Qui test and the Fisher test were used with a statistic significance of $5 \%$. Results: it was observed the parafuntional habit was presented in $50 \%$ of the cases with temporomandibular dysfunction. It wasn't possible a relationship between bruxism and the level of symptoms in temporomandibular jaw dysfunction, by Anamnesic Index. Conclusion: the results of this study suggest that subjects with symptoms of temporomandibular jaw dysfunction must be asked and evaluated about bruxism, regardless of the level of temporomandibular dysfunction. Besides this, it is important to investigate the diagnosis and the treatment of bruxism also in patients without temporomandibular jaw dysfunction in order to prevent development of lesions in the temporomandibular joints and the other structures of the stomatognatic system.

KEYWORDS: Bruxism; Facial Pain; Temporomandibular Joint Dysfunction Syndrome

\section{REFERÊNCIAS}

1. Okeson J. Tratamento das desordens temporomandibulares e oclusão. 4. ed. São Paulo: Artes Médicas; 2000.

2. Dekon SF, Pellizzer EP, Zavanelli AC, Ito L, Rezende CA. Reabilitação oral em paciente portador de parafunção severa. Rev Odonto. 2003 jan-jul; 24(1):54-9.

3. Soares ISQ, Miranda AFV, Ferreira VJA, Di Nino CQMS. Bruxismo: desempenho da mastigação em adultos jovens. Rev. CEFAC. 2004; 6(4):358-62.

4. Rosa RS. Prevalência de desordens temporomandibulares em universitários e sua associação com fatores oclusais, articulares e bruxismo [tese]. Campinas (SP): Universidade Estadual de Campinas; 2004.

5. Zarb GA, Carlsson GE, Sessle BJ, Mohl ND. Disfunções da articulação temporomandibular e dos músculos da mastigação. 1. ed. São Paulo: Santos; 2000.

6. Ciancaglini R, Gherlone EF, Radaelli G. The relationship of bruxism with craniofacial pain and symptoms from the masticatory system in the adult population. J Oral Rehabil. 2001; 28(9):842-8.

7. Glaros AG, Williams K, Lausten L. The role of parafunctions, emotions and stress in predicting facial pain. J Am Dent Assoc. 2005; 136(4):451-8.
8. Matheus RA, Ghelardi IR, Vega Neto DB, Tanaka EE, Almeida SM, Matheus AF. A relação entre os hábitos parafuncionais e a posição do disco articular em pacientes sintomáticos para disfunção temporo-mandibular. Rev Bras Odontol. 2005; 62(1/2):9-12.

9. Koyano K, Tsukiyama Y, Ichiki R, Kuwata $\mathrm{T}$. Assessment of bruxism in the clinic. $\mathrm{J}$ Oral Rehabil. 2008 Mar; 35(7):495-508. http://dx.doi. org/10.1111/j.1365-2842.2008.01880.x

10. Bianchini EMG. Articulação temporomandibular e fonoaudiologia. In: Ferreira LP, Befi-Lopes DM, Limongi SCO. Tratado de fonoaudiologia. São Paulo: Roca; 2004. p. 315-29.

11. Bove SRK, Guimarães AS, Smith RL. Caracterização dos pacientes de um ambulatório de disfunção temporomandibular e dor orofacial. Rev Latino-am Enferm. 2005 set-out; 13(5):686-91. 12. Manfredi APS, Bortolleto PPB, Silva AA, Araújo IEM, Araújo S, Vendite LL. Environmental stress and temporomandibular disorder (TMD) among members of a public university in Brazil. Braz $\mathrm{J}$ Oral Sci. 2006; 5(18):1074-8.

13. Martins RJ, Garcia AR, Garbin CAS, Sundefeld MLMM. Relação entre classe socioeconômica e fatores demográficos na ocorrência da disfunção temporomandibular. Cienc. Saúde Coletiva. 2008; 13(Supl2):2089-96. 
14. Toledo BAS, Capote TSO, Campos JADB. Associação entre disfunção temporomandibular e depressão. Cienc Odontol Bras. 2008; 11(4):75-9.

15. Ćelić R, Jerolimov V, Pandurić J, Haban V. Depression and somatization in patients with temporomandibular disorders. Acta Stomatol Croat. 2006 Jan; 40(1):35-45.

16. Martins RJ, Garcia AR, Garbin CAS, Sundefeld MLM. Associação entre classe econômica e estresse na ocorrência da disfunção temporomandibular. Rev Bras Epidemiol. 2007; 10(2):215-22.

17. Bretan O, Nogueira EA. Distúrbios temporomandibulares e alterações da musculatura mastigatória. Arq Int Otorrinolaringol. 2005; 9(2):140-4.

18. Lobbezoo $\mathrm{F}$, van der Zaag J, van Selms MK, Hamburger HL, Naeije M. Principles for the management of bruxism. J Oral Rehabil. 2008; 35(7):509-23.

19. Fonseca DM, Bonfante G, Valle AL, Freitas SFT. Diagnóstico pela anamnese da disfunção craniomandibular. Rev Gauch Odontol. 1994; 42(1):23-8.

20. Dworkin SF, LeRsche L. Research diagnostic criteria for temporomandibular disorders: review, criteria, examinations and specifications, critique. $J$ Craniomandib Disord. 1992; 6(4):301-55.

21. Branco RS, Branco CS, Tesch RS, Rapoport A. Frequência de relatos de parafunções nos subgrupos diagnósticos de DTM de acordo com os critérios diagnósticos para pesquisa em disfunções temporomandibulares (RDC/TMD). Dent Press Ortodon Ortop Facial. 2008 mar-abr; 13(2):61-9.

22. Van Selms MKA, Lobbezoo F, Visscher CM, Naeije M. Myofascial temporomandibular disorder pain, parafunctions and psychological stress. J Oral Rehabil. 2008; 35(1):45-52. http://dx.doi. org/10.11111/j.1365-2842.2007.01795.x

23. Areias MEQ, Guimarães LAM. Gênero e estresse em trabalhadores de uma universidade pública do estado de São Paulo. Psicol Estudo. 2004 mai-ago; 9(2):255-62.

24. Molina OF, Mazzetto M, Zaccani CB, Mainieri ET, Sobreira M, Rezende SM. A disfunção mastigatória e o "desconforto dental" em três grupos definidos de pacientes com bruxismo e DCM - comparação com um grupo controle. J Bras Ortodon Ortop Facial. 2000 mai-jun; 5(27):51-65.

RECEBIDO EM: 20/07/2009

ACEITO EM: 06/11/2009

Endereço para correspondência:

Geovana de Paula Bolzan

Rua Major João Scherer, 177

Santa Maria - RS

CEP: 97095-410

E-mail: gebolzan@gmail.com 